\title{
Spectrum of Lymph Node Lesions by Fine Needle Aspiration Cytology: A Retrospective Analysis
}

\author{
Shilpa Somashekar Biradar ${ }^{1}$ and Deepa Siddappa Masur ${ }^{2}$ * \\ ${ }^{1}$ Dept. of Pathology, Akash Institute of Medical Sciences and Research Centre, Devanahalli, Bangalore, Karnataka, India \\ ${ }^{2}$ Dept. of Pathology, S. N. Medical College, Bagalkot, Karnataka, India
}

\begin{abstract}
Background: Fine needle aspiration cytology (FNAC) is a veritable tool for the assessment and diagnosis of superficial lymph node enlargement. The cytologic patterns of lymph node fine needle aspirations exhibit a wide variation in different diseases. Lymphadenopathy is of great clinical significance and the underlying cause may range from a treatable infectious etiology to malignant neoplasms. The aim of the present study is to study and evaluate the patterns of various lymph node lesions on fine needle aspiration cytology

Methods: This retrospective study was conducted on 160 selected patients including all age groups and both sexes with lymphadenopathy who had undergone FNAC. We reviewed all the cases of lymphadenopathies. The cytomorphological features seen in the aspirate were critically analysed and correlated with their aetiology

Result: Out of 160 cases, the most frequent cause of lymphadenopathy was found to be Reactive Lymphadenitis with 89 cases (55.62\%). The next frequent diagnosis was Tuberculosis with 38 cases $(23.75 \%)$ followed by malignant lymphoma in 8 cases $(5 \%)$ and metastatic lymphadenopathy in 7 cases $(4.37 \%)$.
\end{abstract}

Conclusion: FNAC is a simple, safe, reliable, and inexpensive method in early detection of lymph node lesions, which has been proven in this study again.

\section{Keywords: Fine Needle Aspiration Cytology, Lymphadenopathy, Tuberculous Lymphadenitis, Reactive Lymphadenitis}

\section{Introduction}

Fine needle aspiration cytology was thought as a means to confirm a clinical suspicion of local recurrence or metastasis of known cancer without subjecting the patient to further surgical intervention. The role of FNAC is not limited to neoplastic conditions. It has a valuable role in the diagnosis of inflammatory, infectious and degenerative conditions. The technique is minimally invasive and gives a speedy result ${ }^{[1]}$.Lymph nodes are an important part of the immune system. Lymph nodes become enlarged in a wide spectrum of diseases, including infection and malignancy. Cytological examination of FNA smears can determine whether lymphadenopathy is due to reactive hyperplasia, infection, metastatic malignancy or malignant lymphoma ${ }^{[2]}$. The present study reports the results of FNAC of lymphadenopathies based on cytomorphology.

\section{Materials and Methods}

This was a retrospective study conducted in a tertiary hospital for a period of one year. The study was conducted on 160 patients including all age groups and both sexes who underwent FNAC for palpable lymphadenopathy, either single or multiple.
A brief clinical history followed by physical examination was done and the findings were noted. FNAC was performed under aseptic precautions using 22-24 Gauge needles attached to $10 \mathrm{ml}$ syringes. The aspirated material was smeared on to the glass slides. The smears were then fixed in 95\% ethyl alcohol and stained with Hematoxylin and Eosin stain and Papanicolaou stain. Leishman stain was done on air dried smears. Zeil-Neelson staining was done whenever required. The cytological diagnosis for each case was based on cytomorphology and available clinical information

\section{Result}

A total number of 160 patients were studied. Among them, $82(51.25 \%)$ patients were male and $78(48.75 \%)$ were female patients(Table 1). The age of the patients ranged from 3 months to 80 years (Table 2). Cervical lymph nodes were enlarged in 111 of 160 cases $(69.37 \%)$ followed by submandibular lymph nodes in 17 cases $(10.62 \%)$, supraclavicular lymph nodes in 10 cases $(6.25 \%)$, axillary in 8 cases $(5 \%)$, inguinal in 5 cases $(3.12 \%)$, submental in 4 cases $(2.5 \%)$ (Table 3). Multiple lymph nodes were involved in 5 cases $(3.12 \%)$. Five cases had inadequate material and were thus unsatisfactory for 
evaluation. Among 160 cases, maximum number of cases were recorded in age group less than 20 years. A major proportion of lymphadenopathies in this study were due to benign conditions $(90.62 \%)$.Out of 160 cases, the most frequent cause of lymphadenopathy was found to be Reactive Lymphadenitis with 89 cases (55.62\%). The next frequent diagnosis was Tuberculosis (Figure 1) with 38 cases $(23.75 \%)$ followed by malignant lymphomas (Figure $2)$ in 8 cases $(5 \%)$ and metastatic lymphadenopathy in 7 cases $(4.37 \%)$. Acute suppurative lymphadenitis was seen in 7 cases $(4.37 \%)$ and granulomatous lymphadenitis in 6 cases(3.75\%) (Table 4).

Table 1: Gender wise distribution of patients $(n=160)$

\begin{tabular}{|c|c|c|}
\hline \multirow{2}{*}{ Male } & Number of cases & Percentage \\
\cline { 2 - 3 } & 82 & $51.25 \%$ \\
\hline Female & 78 & $48.75 \%$ \\
\hline
\end{tabular}

Table 2: Age wise distribution of patients $(n=160)$

\begin{tabular}{|c|c|c|}
\hline Age group in years & Number of cases & Percentage \\
\hline $0-20$ & 75 & 46.87 \\
\hline $21-40$ & 60 & 37.5 \\
\hline $41-60$ & 17 & 10.62 \\
\hline $61-80$ & 08 & 5 \\
\hline
\end{tabular}

Table 3: Sites of lymph node involvement $(n=160)$.

\begin{tabular}{|c|c|c|}
\hline Site & Number of cases & Percentage \\
\hline Cervical & 111 & $69.37 \%$ \\
\hline Axillary & 08 & $5 \%$ \\
\hline Supraclavicular & 10 & $3.25 \%$ \\
\hline Inguinal & 05 & $12 \%$ \\
\hline Submandibular & 17 & $2.5 \%$ \\
\hline Sultiple lymph nodes & 04 & $3.12 \%$ \\
\hline
\end{tabular}

Table 4: Cytological diagnoses of lymph node aspirations $(n=160)$.

\begin{tabular}{|c|c|c|}
\hline Diagnosis & Number of cases & Percentage \\
\hline Reactive Lymphadenitis & 89 & $55.62 \%$ \\
\hline Tubercular Lymphadenitis & 38 & $23.75 \%$ \\
\hline Malignant Lymphoma & 09 & $5.62 \%$ \\
\hline Metastatic Lymphadenopathy & 07 & $4.37 \%$ \\
\hline Acute Suppurative Lymphadenitis & 06 & $3.75 \%$ \\
\hline Granulomatous Lymphadenitis & 06 & $3.75 \%$ \\
\hline Inadequate & 05 & $3.12 \%$ \\
\hline
\end{tabular}

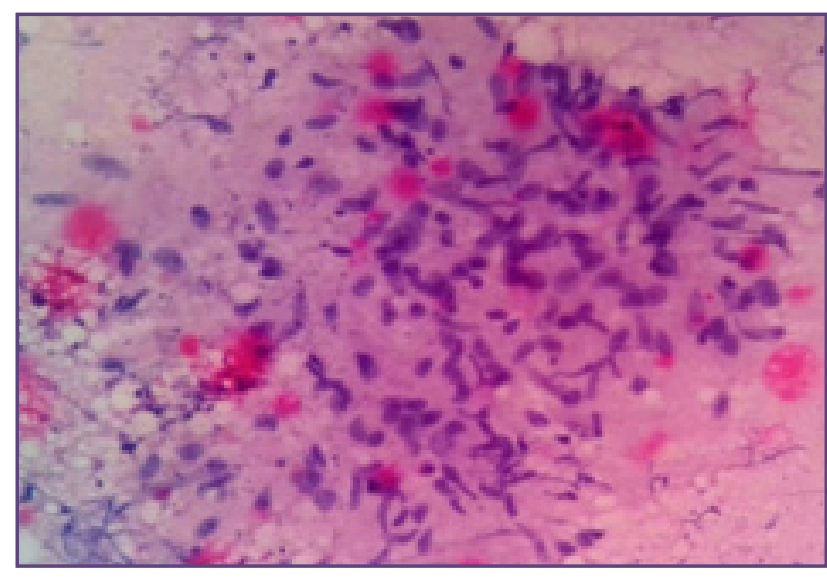

Fig. 1: Smear shows epithelioid cell granulomas and necrosis in the background (H \& E stain ;400x).

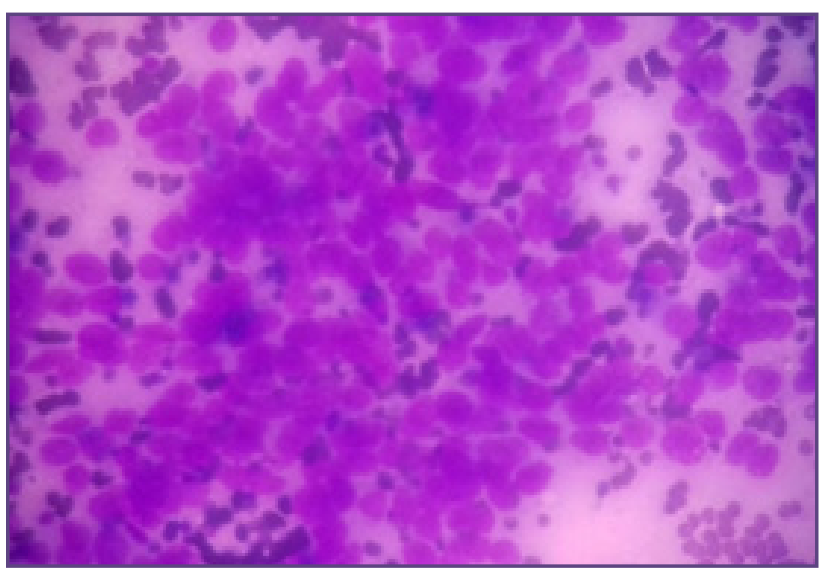

Fig. 2: Smear shows clusters of atypical lymphoid cells from axillary lymph node FNAC (PAP Stain;400x). 


\section{Discussion}

FNAC is the study of cellular aspirate obtained through a fine needle under negative pressure. The technique is relatively painless and economical. It can give unequivocal diagnosis in most of the conditions. FNAC is a simple, quick, and inexpensive procedure that is used to sample superficial masses. The procedure is performed in the outpatient clinic. Lymphadenopathy is an abnormal increase in size and altered consistency of lymph nodes. It is a clinical manifestation of regional or systemic disease and serves as an excellent clue to the underlying disease. In some cases, cervical lymphadenopathy may be the only clinical finding. This can be a clue for many underlying clinical conditions ${ }^{[3]}$. Our study highlights the spectrum of cytological findings of various lymphadenopathies on fine needle aspiration cytology.

Majority of lymphadenopathies in this study were due to benign conditions $(90.62 \%)$, which was in accordance with an earlier study, in which $86.4 \%$ of the lesions were benign ${ }^{[4]}$. The study conducted by Tilak ${ }^{[5]}$ et al revealed that the lesion arising in lymph nodes can be found in patients of different age groups, ranging from an early to advanced age. This was correlated with our findings where we found that the youngest patient in the present study was 3 months old and the oldest one was 80 years old. These figures came in close comparison to other studies. In the present study, $75(46.87 \%)$ patients were in the age group of $0-20$ years. Simlar to the observation of Gupta et al $52.26 \%{ }^{[6]}$, whereas in the study of Pandit AA et al ${ }^{[7]}$, most of the patients $146(51.05 \%)$ were in the age group of 21-40 years. As compared to other studies, male predominance was noted in the current study. These findings are comparable with studies conducted by Gupta et al ${ }^{[6]}$ and Khajuria et al ${ }^{[8]}$. The present study revealed that the most common group of lymph node involved were cervical nodes which is in accordance with other studies done by Kochhar et al ${ }^{[9]}$, Pavithra et al ${ }^{[10]}$.In the current study, Reactive lymphadenitis was the most common lesion and was reported in $55.62 \%$ cases. This result was comparable to other studies, where its incidence ranged from $18.9 \%$ to $42 \%$ [8,9,11,12,13,14 .The second common diagnosis in the present study was Tuberclar lymphadenitis accounting to $23.75 \%$ of cases. Low incidence of AFB positivity on ZN smears was noted in our study (15\%) which is in accordance with the study done by Agarwal et a ${ }^{[15]}(19.65 \%)$. This could be attributed to the compromised immune status or inadequacy of the cellular immune response. In this study, we considered the presence of scattered epithelioid cells with or without granulomas or only necrotic material with neutrophilic infiltration as tuberculous lymphadenitis, inspite of AFB being absent in these smears ${ }^{[16]}$. Granulomatous lymphadenopathies constituted $3.75 \%$ of cases. Acute suppurative lymphadenopathy was observed in $4.37 \%$ cases in our study which is in accordance with other studies done by Kochhar et al (4\%) and Patra et al ${ }^{[17]}$ (5.8\%).Malignant lymphomas were less in our study constituting $5.62 \%$ of all the cases. Similar observations were seen in other studies ${ }^{[18,19,12,8]}$. Non-Hodgkin's Lymphoma was reported in $5(3.12 \%)$ out of 9 lymphoma cases whereas $4(2.49 \%)$ cases of Hodgkin's lymphoma were reported. Lymph node aspirates in $4.37 \%$ cases showed metastatic deposits predominantly squamous cell carcinoma. Similar results were obtained in the study conducted by Pavithra et al ${ }^{[10]}$ .In the current study $3.12 \%$ of cases were unsatisfactory to report due to low cellular yield.

Despite the limitations, FNAC provides a simple, reliable and convenient method for the initial management of cervical lymphadenopathy. FNAC has a valuable role in diagnosing neoplastic and metastatic lesions. It helps in detecting metastatic diseases and also gives the clue regarding the origin of the primary tumor.

\section{Conclusion}

FNAC is a very important diagnostic tool for diagnosing benign as well as malignant lesions. It is a simple, safe and inexpensive definite diagnostic procedure to render a diagnosis, especially in lymph node aspirates. Our study highlighted the various cytomorphological patterns of lymphadenopathies. We conclude that,the benign results should be interpreted in the context of clinical findings and if clinical malignancy is highly suspected, further evaluation is justified.

\section{Reference}

1. Orell SR, Sterrett GF, Fine needle Aspiration Cytology.5th edition. Churchill Livingstone. 2012

2. Bibbo M, Comprehensive Cytopathology. 2nd edition.W.B.Saunders Company.1991

3. Kataria P, Sachdeva M, Singh NK. FNAC as a diagnostic tool for the diagnosis of cervical lymphadenopathy. Bull Environ Pharmacol Life Sci 2012;1:72-5.

4. Ahmad S, Akhtar S, Akhtar K, Naseem S, Mansoor T. Study of Fine needle aspiration cytology in lymphadenopathy with special reference to acid-fast staining in cases of tuberculosis. JK science 2005;7 (1):1-4.

5. Tilak V, Dhadel AV, Jain R. Fine needle aspiration cytology of the head and neck masses. Ind $\mathrm{J}$ Pathol Microbiol.2002;45(1):23-30.

6. Gupta AK, Nayar M, Chandra M. Reliability and limitations of fine needle aspiration cytology of lymphadenopathies. An analysis of 1,261 cases. Acta Cytol 1991;35:777-83.

7. Pandit AA,Candes FP, Khubchandani SR. Fine needle aspiration cytology of lymph nodes.J Postgrad Med.1987;33(3):134-6 
8. Khajuria R, Goswami KC, Singh K, Dubey VK. Pattern of lymphadenopathy on FNAC in Jammu. JK Sci 2006;8:157-9.

9. Kochhar AK, Duggal G, Singh K, Kochhar SK. Spectrum of cytological findings in patients with lymphadenopathy in rural population of South Haryana, India - Experience in a tertiary care hospital. Internet J Pathol.2012;13(2):8

10. Pavithra P, Geetha JP.Role of fine needle aspiration cytology in the evaluation of the spectrum of lymph node lesions. Int J Pharm Bio Sci. 2014;5(4):377-384

11. Mohanty R, Wilkinson A. Utility of fine needle aspiration of lymph nodes. IOSR J Dent Med Sci.20138(5);13-8.

12. Hirachand S, Lakhey M, Akhter J, Thapa B. Evaluation of fine needle aspiration cytology of lymph nodes in Kathmandu Univ Med J.2009;7:139-42.

13. Adhikari P, Sinha BK,Baskota DK. Comparison of fine needle aspiration cytology and histopathology in diagnosing cervical lymphadenopathies. Australas Med J.2011;4:97-9.

14. Mallick D, Nathprasad R, Gon S, Ghosh G.Spectrum of Lymph Node Lesions by fine needle aspiration cytology in worker population of Eastern zone of India. IJOH.2015;7(3):139-144.

15. Aggarwal P, Wali JP, Singh S, Handa R, Wig N, Biswas A. A clinicobacterial study of peripheral tuberculous lymphadenitis.J Assoc Physicians India. 2001; 49: 808-12.

16. Paul PC, Goswami BK, Chakrabarti S, Giri A, Pramnik R. Fine needle aspiration cytology of lymphnodes - an institutional study of 1448 cases over a five year period.J Cytol.2004;21:187-190.

17. Patra AK, Nanda BK, Mahapatra BVK, Panda AK. Diagnosis of lymphadenopathy by fine needle aspiration cytology. Indian J Pathol Microbiol.1983;26 :272-8.

18. Annam V, Kulkarni MH, Puranik RB. Clinicopathologic profile of significant cervical lymphadenopathy in children aged 1-12 years. Acta Cytol 2009;53:174-8.

19. Fatima S,Arshad S, Ahmed Z, Hasan SH.Spectrum of cytological findings in patients with neck lymphadenopathyexperience in tertiary hospital in Pakistan. Asian Pac J Cancer Prev.2011;12:1873-5.

*Corresponding author:

Dr. Deepa S Masur\#Assistant Professor, Dept. Of Pathology, S.N.Medical College, Bagalkot, Karnataka, India

Email: drdns11s@gmail.com

Date of Submission : 13.02.2017

Date of Acceptance : 03.03.2017

Financial or other Competing Interests: None.
Date of Publication : 14.06.2017 